\title{
Strongly Correlated States of Ultracold Rotating Dipolar Fermi Gases
}

\author{
Klaus Osterloh, ${ }^{1}$ Nuria Barberán, ${ }^{2}$ and Maciej Lewenstein ${ }^{3}$ \\ ${ }^{1}$ Institute for Theoretical Physics, University of Hannover, Appelstr. 2, D-30167 Hannover, Germany \\ ${ }^{2}$ Dept. ECM, Facultat de Física, Universitat de Barcelona, E-08028 Barcelona, Spain \\ ${ }^{3}$ ICFO-Institut de Ciències Fotòniques, Parc Mediterrani de la Tecnologia, Spain
}

(Received 7 March 2007; published 17 October 2007)

\begin{abstract}
We study strongly correlated ground and excited states of rotating quasi-2D Fermi gases constituted of a small number of dipole-dipole interacting particles with dipole moments polarized perpendicular to the plane of motion. As the number of atoms grows, the system enters an intermediate regime, where ground states are subject to a competition between distinct bulk-edge configurations. This effect obscures their description in terms of composite fermions and leads to the appearance of novel quasihole ground states. In the presence of dipolar interactions, the principal Laughlin state at filling $\nu=1 / 3$ exhibits a substantial energy gap for neutral (total angular momentum conserving) excitations and is well-described as an incompressible Fermi liquid. Instead, at lower fillings, the ground state structure favors crystalline order.
\end{abstract}

PACS numbers: $03.75 . S s, 73.43 .-\mathrm{f}$

Some of the most fascinating challenges of modern atomic and molecular physics arguably concern ultracold dipolar quantum gases [1]. The recent experimental realization of a quantum degenerate dipolar Bose gas of Chromium [2] and the progress in trapping and cooling of dipolar molecules [3] have opened the path towards ultracold quantum gases with dominant dipole interactions [4]. Particularly interesting in this context are rotating dipolar gases (RDG). Novel forms of vortex lattices, e.g., square, stripe- and bubble-"crystal" lattices are expected to occur in Bose-Einstein condensates of RDGs [5]. The stability of these phases in the lowest Landau level was recently investigated [6]. We have shown that the quasihole gap survives the large $N$ limit for fermionic RDGs [7]. This suggests that the same occurs for angular momentum conserving particle-hole excitations, and makes RDGs perfect candidates to approach the strongly correlated regime and to realize Laughlin liquids (cf. [8]) at filling $\nu=1 / 3$, and quantum Wigner crystals at $\nu \leq 1 / 7$ [9] for a mesoscopic number of atoms $N \simeq 50-200$. Lately, Rezayi et al. [10] have shown that the presence of a small amount of dipoledipole interactions stabilizes the so-called bosonic RezayiRead state at $\nu=3 / 2$ whose excitations are both fractional and non-Abelian.

In this Letter, we investigate quasi-2D microscopic and mesoscopic clouds of fermions whose dipole moments are polarized perpendicular to their plane of motion. Results are obtained by exact diagonalization of the Hamiltonian of the system for $N=3-12$ particles. Such systems can be realized with arrays of rotating microtraps, or optical lattices with rotating on-site potential wells in $1 \mathrm{D}, 2 \mathrm{D}$, or 3D, or using a 1D lattice rotating around its axis. Such systems, consisting indeed of multiple copies of small atomic clouds that facilitate detection, are of great interest recently (see [11] and references therein). Here, we study the lowest Landau level (LLL) physics, in particular, the crossover from the weakly interacting Fermi liquid regime to strongly correlated fractional quantum Hall-like states.
As $N$ grows, particles either organize themselves in the bulk or crystallize on the edge. This competition is based on interparticle correlations and exceeds the scope of the effective theory of composite fermions [12]. It leads to the appearance of novel ground states (GS's) with a quasihole at the origin which are linked to unfavored compositefermion states. This surprising behavior neither occurs for Van der Waals nor for Coulomb forces with $N \geq 10$ and is the main result of this Letter. The analysis of the principal Laughlin state at filling $\nu=1 / 3$ reveals that its low energy excitations in the angular momentum subspaces where quasiholes are to be found correspond to edge excitations rather than topological quasihole defects. Instead, "neutral" particle-hole pair excitations are already substantially gapped in these microscopic systems.

We consider a system of $N$ dipolar fermions rotating in an axially symmetric harmonic trapping potential strongly confined in the direction of the axis of rotation. Along this $z$-axis, the dipole moments, as well as spins, are assumed to be aligned. In case of low temperature $T$ and weak chemical potential $\mu$ with respect to the axial confinement $\omega_{z}$, the gas is effectively $2 \mathrm{D}$, and the Hamiltonian of the system in the rotating reference frame reads

$$
\mathcal{H}=\sum_{j=1}^{N} \frac{1}{2 M}\left(\vec{p}_{j}-M \Omega \overrightarrow{\mathbf{e}}_{z} \times \vec{r}_{j}\right)^{2}+\frac{M}{2}\left(\omega_{0}^{2}-\Omega^{2}\right) r_{j}^{2}+V_{d} .
$$

Here, $\omega_{0} \ll \omega_{z}$ is the radial trap frequency, $\Omega$ is the frequency of rotation, $M$ is the mass of the particles, $V_{d}=$ $\sum_{j<k}^{N} \frac{d^{2}}{\left|\vec{r}_{j}-\vec{r}_{k}\right|^{3}}$ is the dipolar interaction potential (rotationally invariant with respect to the $z$-axis), $d$ is the dipole moment, and $\mathbf{r}_{j}=x_{j} \mathbf{e}_{x}+y_{j} \mathbf{e}_{y}$ is the position vector of the $j$-th particle. The first term of (1) is formally equivalent to the Landau Hamiltonian of particles with mass $M$ and charge $e$ moving in a constant magnetic field of strength $B=2 M \Omega c / e$ perpendicular to their plane of motion. The 


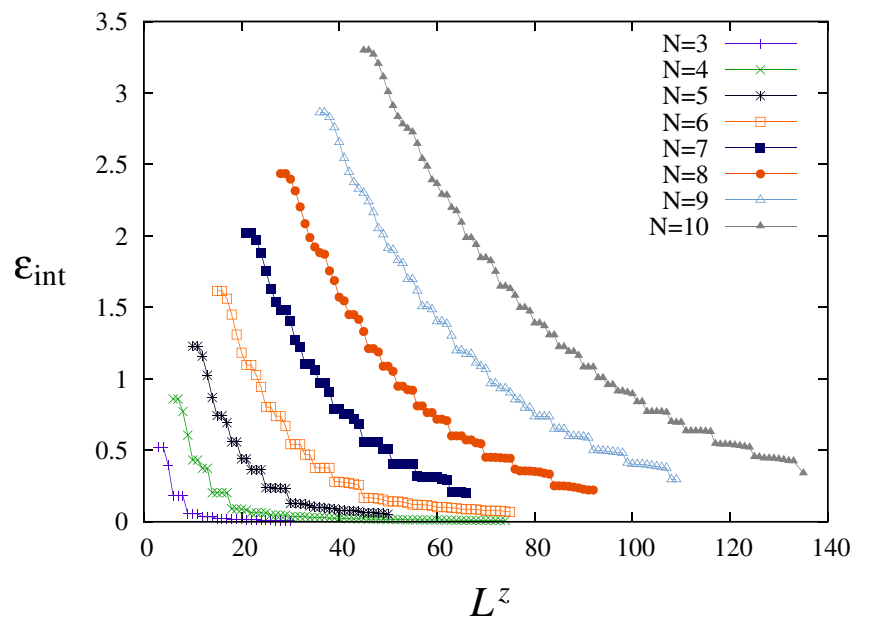

FIG. 1 (color online). Interaction contribution to the GS energy as a function of $L^{z}$.

eigenvectors of $\mathcal{H}_{\text {Landau }}$ span Landau levels (LL) with energies $\varepsilon_{n}=\hbar \omega_{c}(n+1 / 2)$ where $\omega_{c}=2 \Omega$. We denote by $N_{\mathrm{LL}}=1 / 2 \pi l^{2}$ the number of states per unit area in each LL, where $l=\sqrt{\hbar / M \omega_{c}}$ is the magnetic length. Given a fermionic density $n_{f}$, the filling factor $\nu=2 \pi l^{2} n_{f}$ refers to the fraction of occupied LLs. Even though the above definition applies to infinite homogeneous systems, it may be used for finite systems using suitable truncation of the Hilbert space at specific angular momenta. The second term in (1) accounts for a rotationally induced effective reduction of the trap strength. For $\Omega \rightarrow \omega_{0}$, the confining potential vanishes.

In the following, it is assumed that particles solely occupy the LLL. In the LLL Hamiltonian, there are two competing contributions to the energy: the kinetic term given by $\hbar\left(\omega_{0}-\Omega\right) \hat{L}_{z}$, and the interaction term, which scales as $d^{2} / l^{3}=2 \hbar \omega_{0}\left(a_{d} / l\right)$ with $a_{d}=M d^{2} / \hbar^{2}$. The natural unit of energy is $\hbar \omega_{0}$, whereas distances are measured in $l$; from now on, we set $a_{d} / l \equiv 1$. We analyze the ground state interaction energy for a given $L^{z}$. It reveals plateaus, clearly visible for small $N$ (see Fig. 1).

Ground state candidates are the first states of these plateaus where a downward cusp occurs in the spectrum.

By tuning the rotational frequency, some of these states are selected as true ground states at specific "magic" angular momenta as depicted in Fig. 2. For relatively low values of $\Omega$, the ground state is the filled LL state at $\nu=1$, which is insensitive to type and strength of interaction as long as the LLL approximation holds. When $\Omega$ is continuously increased, the system evolves from the weakly interacting (where the kinetic term is dominant) to strongly correlated regime (where the interaction is dominant). In case of short-range interactions, this process terminates at $L_{0}^{z}=\nu_{0}^{-1} N(N-1) / 2$, where the fermionic (bosonic) Laughlin states at filling $\nu_{0}=1 / 3(1 / 2)$ become the true ground states. The existence of a final $L^{z}$ is due to the fact that the ground state contact interaction energy vanishes for $L^{z} \geq L_{0}^{z}$. The long range nature of dipolar interactions

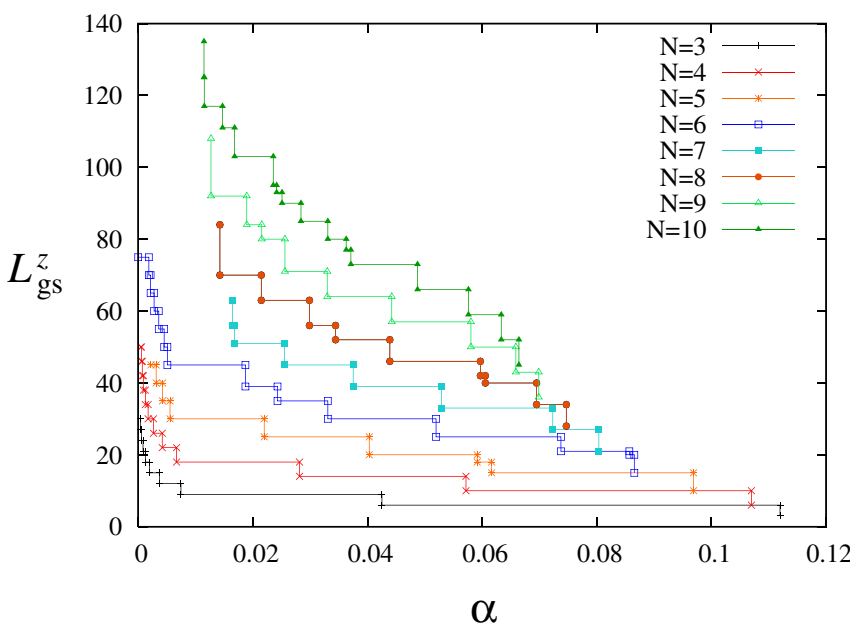

FIG. 2 (color online). Ground state angular momentum series over $\alpha \equiv \omega_{0}-\Omega$ (the divergence at $\alpha=0$ is not shown).

lifts this degeneracy. Thus, the whole principal series of fillings, i.e., $\nu=1 /(2 m+1)$ for fermionic gases, is accessible. To reveal the internal structures of relevant states, we consider the density-density correlation function $\hat{\rho}\left(\vec{r}, \vec{r}_{0}\right)$, which represents the conditional probability to find one atom at $\vec{r}$ when another is simultaneously located at $\vec{r}_{0}$,

$$
\hat{\rho}\left(\vec{r}, \vec{r}_{0}\right)=\sum_{j<k}^{N} \delta\left(\vec{r}_{j}-\vec{r}\right) \delta\left(\vec{r}_{k}-\vec{r}_{0}\right) .
$$

It is crucial to analyze second order correlations since the GS density only reveals radially symmetric contributions. Figure 3 depicts $\hat{\rho}\left(\vec{r}, \vec{r}_{0}\right)$ for a selection of ground states with $N=10$ particles.

Generally, for small $N$, starting from the unstructured maximum-density droplet at $L^{z}=N(N-1) / 2$ (top left)

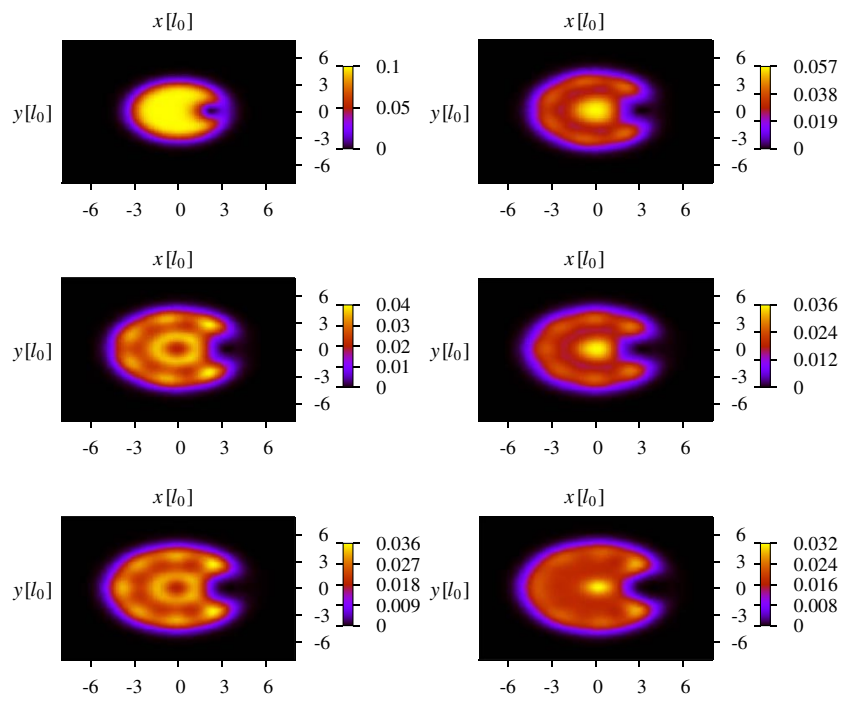

FIG. 3 (color online). Ground state density-density correlation functions $\hat{\rho}\left(\vec{r}, \vec{r}_{0}\right)$ for $N=10$ dipolar fermions at $L^{z}=45,80$ (top); 90, 93 (centered); 103, 117 (bottom) with $\vec{r}_{0}$ set to the maximum of the density, which occurs at the edge. 
with $\nu=1$, a fraction of atoms starts to arrange itself in a crystal on the edge leaving a residual "Fermi sea" at the center (top right), until the correlations are homogeneously established at $L_{0}^{z}=3 N(N-1) / 2$. For larger $N$, however, the energetically favorable number of edge atoms in the crystal changes irregularly with $L^{z}$ (right column); the system seems to be frustrated in this respect. In this regime, the bulk has to reorganize itself accordingly, and novel fascinating states with a hole at the center of the bulk appear (centered and bottom left).

In order to understand the magic $L^{z}$ numbers, Jain et al. [12] have proposed to model the interacting system in terms of an effective theory of noninteracting composite fermions (CF) for electrons in a quantum dot. In this ansatz, each fermion captures an even number of quantum fluxes, and the wave function reads

$$
\Psi_{\mathrm{CF}}\left(\left\{z_{j}\right\}\right)=\mathcal{N} \hat{\mathcal{P}}_{\text {LLL }}\left\{\prod_{j<k}^{N}\left(z_{j}-z_{k}\right)^{2 m} \Psi_{\text {Landau }}\right\} .
$$

Here, $m$ denotes the number of flux pairs, $\Psi_{\text {Landau }}$ is an $N$-particle eigenfunction of $\mathcal{H}_{\text {Landau }}$, and $\hat{\mathcal{P}}_{\text {LLL }}$ is the LLL projector. Cooper and Wilkin have nicely adapted this scheme to bosonic contact interacting gases [13].
Following this idea, we compared the series of true GS for noninteracting composites and dipolar fermions. As long as there is no real bulk in the system, i.e., for $N<$ 7 , the ground state series nearly identically matches with the predictions of the effective CF theory. Furthermore, overlap calculations for electrons and short-range interacting bosons $[12,13]$ suggest that similar results will hold for dipolar fermions. However, for bigger $N$, deviations from $\mathrm{CF}$ theory, in particular, in the intermediate regime, occur [see, for instance, the magic numbers for CF GS's, and true magic numbers for exact GS's in Table ()]. This deficiency of the CF theory, already commented on in Ref. [13], is more clearly related to the frustration effect and reorganization of the bulk in the present case. For $N=10$, ground states with a density defect at the center are found at $L^{z}=$ 90,95 , and 103. They either have no CF counterpart $\left(L^{z}=\right.$ 95), or are hardly similar to the corresponding CF states. Instead, the analysis for the accessible range of systems strongly suggests that these states originate from parent states which are boosted by $N$ quanta of angular momentum, e.g., $L^{z}=80,85$, and 93 for the above series. None of these parent states has its magic analogues in the $\mathrm{CF}$ model.

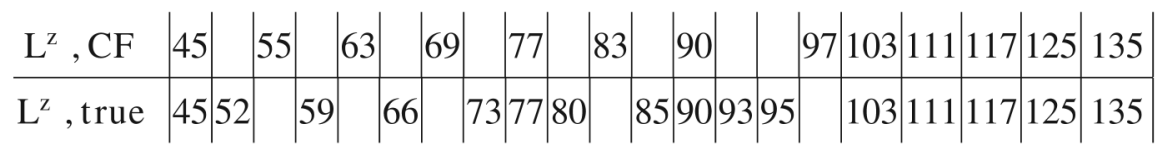

To a good approximation, the close connection between parent and boosted state (e.g., $L=93$ and 103) can be understood as a quasihole $(\mathrm{QH})$ excitation. This is analytically represented by

$$
\Psi_{\mathrm{qh}}\left(\left\{z_{j}\right\}\right)=\mathcal{N}\left\{\prod_{j=1}^{N}\left(z_{j}-z_{\mathrm{c.m}}\right)^{m} \Psi_{L^{z}}\right\}
$$

where $z_{\text {c.m. }}=\left(\sum_{j=1}^{N} z_{j}\right) / N$ and $m=1$. Indeed, the above wave function proves to be a good approximation for the states with $L^{z}=L_{\text {parent }}^{z}+N$. The states (5), similarly as the exact GS's, reveal a "smoothed" topological defect at the origin due to finite size. The density of the exact GS's at the origin scales as $1 / N$ and decreases from 0.14 to $\simeq 0.10$ as $N$ varies from 7 to 11 , while their overlaps with the states (5) grow from 0.6 to 0.7 despite the significant increase of the relevant Hilbert space dimension [14].

We have also studied in detail low energy excitations of the dipolar Laughlin state at $\nu=1 / 3$, which for $N \geq 10$ consists of a significant bulk, surrounded by a practically "melted crystal" at the edge (see Fig. 4).

In order to relate our results to the ones obtained in the thermodynamical limit in Ref. [7], it is necessary to identify the finite-size analogues of $\mathrm{QH}$ excitations in the spectrum. To map out irrelevant candidates, it is reasonable to appropriately truncate the Hilbert space by a finite-size filling factor. We use the approach of Ref. [15], and fix $\nu$ by imposing the constraint $m_{\nu} \leq(N-1) / \nu$ on the maximum single particle angular momentum in the $N$-particle Fock basis. If the rotational frequency $\Omega$ is tuned to favor $\Psi_{1 / 3}^{L}$ as the true ground state, the lower lying excitations for $L_{1 / 3}^{L}<L^{z}<L_{1 / 3}^{L}+N$ turn out to be gapped by $\Delta \epsilon \simeq 1 / N$. For short-range interacting bosonic systems, these states have been partially identified as edge excitations that carry quanta of angular momentum [16]. To identify the excited states with a quasihole at the center, the ground states at $L_{\mathrm{qh}}^{z}=L_{1 / 3}^{L}+N$ are considered. These states closely follow the lowest energy branch of edge excitations, and should be regarded as such, rather than as QH states. This is strongly supported by the fact that the substantial density dip for $N=3$ continuously vanishes for increasing $N$. Additional information about these edge excitations can

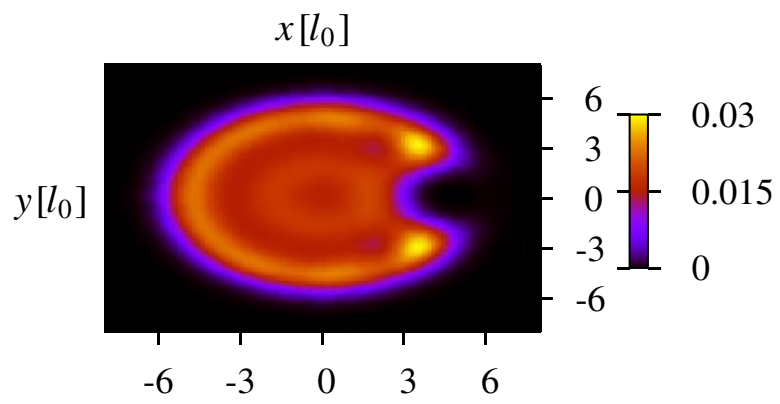

FIG. 4 (color online). Density-density correlation function $\hat{\rho}\left(\vec{r}, \vec{r}_{0}\right)$ of the Laughlin state for $N=12$ dipolar fermions with $\vec{r}_{0}$ chosen at the maximum of the density, which occurs at the edge. 
be obtained by a direct qualitative inspection of Fig. 4. For the Laughlin state, edge excitations are described by a Luttinger liquid theory [17], and their amplitude decays as $\sin ^{-3}(\phi)$, where $\phi$ is the angular distance from $\vec{r}_{0}$ measured along the edge. Here, the decay is much slower, due to finite-size effects.

The above considerations imply that the only reliable quantity that remains to estimate the $\mathrm{QH}$ energy in the large $N$ limit is the neutral gap at fixed $L^{z}$. This gap remains more or less constant for smaller $N$, and gradually increases as the number of bulk particles in the system and $N$ grows. For the data of Ref. [7] $(M=30$ a.m.u., $d=$ $0.5 \mathrm{D}$, and a trap frequency of $2 \pi \times 10^{3} \mathrm{~Hz}$ ), the gap is of the order of percents of $2 \hbar \omega_{0}$, i.e., it is substantial, but several times smaller than the large $N$ value estimated in [7] in the large $N$ limit. Obviously, the lack of true bulk behavior in the investigated mesoscopic samples is responsible for this effect.

In the semiconductor fractional quantum Hall effect, Wigner crystals, i.e., specific charge-density waves, were discussed as competing ground states to Laughlin liquids. The interplay of quantum fluctuations with the interaction energy have proven crystalline Wigner order to be favorable for low enough fillings for electrons. This behavior intuitively changes in the case of dipolar particles as the interaction energy scales differently in the density of the particles. A very recent detailed analysis of this issue has confirmed the stability estimates for Laughlin and Wigner states of Ref. [7] for systems constituted of 50-200 particles [9]. Fehrmann et al. have proven that phonon excitations destabilize the Wigner crystal for fillings $\nu>1 / 7$, which melts into a Laughlin liquid. In the microsystems discussed in this Letter, this crossover is strongly supported. For fillings $\nu \leq 1 / 5$, density profiles significantly deviate from the Laughlin state and show clear signatures of crystalline order for $N=6$ (see Fig. 5).

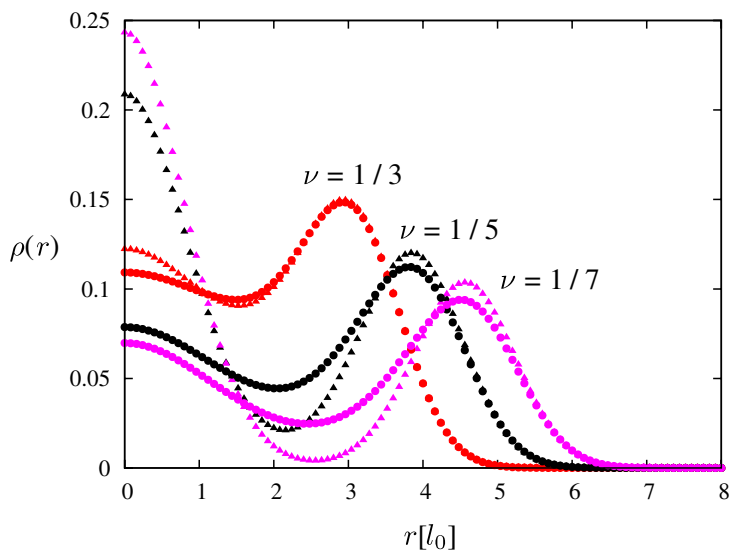

FIG. 5 (color online). Radial density $\rho(r)$ of Laughlin states (circles) and true dipolar ground states (triangles) for $N=6$ at different filling factors. Apart from five particles which constitute the edge ring, localization of the sixth particle at the origin is clearly visible for $\nu \leq 1 / 5$.
Summarizing, we have studied in detail ground and excited states of quasi-2D ultracold rotating dipolar Fermi gases. By exact diagonalization methods, we studied systems up to 12 particles. We have identified novel kinds of strongly correlated states in the intermediate regime, i.e., "boosted" quasihole GS's, which appear alternatively as $\Omega$ grows. Calculation of the substantial gap in the excitation spectrum of the dipolar Laughlin state at $\nu=$ $1 / 3$ prove its accessibility in these microsystems. At lower fillings, interactions favor crystalline order. Rotating dipolar gases are thus very suitable candidates to realize Laughlin-like and more exotic quantum liquids, as well as their crossover behavior to Wigner crystals.

We are indebted to W. Apel for valuable advice and help, and we thank N. Cooper, D. Dagnino, M. Leduc, A. Riera, C. Salomon, L. Sanchez-Palencia, and G. V. Shlyapnikov for helpful discussions. We acknowledge support from the Deutsche Forschungsgemeinschaft Nos. (SPP1116 and SFB 407), ESF PESC “QUDEDIS,", EU IP "SCALA," Spanish MEC Nos. (FIS2004-05639, FIS2005-04627, 2005SGR00343, and Consolider Ingenio 2010 “QOIT”).

[1] M. Baranov, Ł. Dobrek, K. Góral, L. Santos, and M. Lewenstein, Phys. Scr. T102, 74 (2002).

[2] A. Griesmaier, J. Werner, S. Hensler, J. Stuhler, and T. Pfau, Phys. Rev. Lett. 94, 160401 (2005).

[3] Special issue on Ultracold Polar Molecules: Formation and Collisions, edited by J. Doyle, B. Friedrich, R. V. Krems, and F. Masnou-Seeuws [Eur. Phys. J. D 31, 149 (2004)].

[4] T. Lahaye et al., Nature (London) 448, 672 (2007).

[5] N. R. Cooper, E. H. Rezayi, and S.H. Simon, Phys. Rev. Lett. 95, 200402 (2005).

[6] S. Komineas and N. R. Cooper, arXiv:cond-mat/0610702.

[7] M. A. Baranov, K. Osterloh, and M. Lewenstein, Phys. Rev. Lett. 94, 070404 (2005).

[8] The Quantum Hall Effect, edited by R. E. Prange and S. M. Girvin (Springer Verlag, New York, 1987).

[9] M. A. Baranov, H. Fehrmann, and M. Lewenstein, arXiv:cond-mat/0612592.

[10] E. H. Rezayi, N. Read, and N. R. Cooper, Phys. Rev. Lett. 95, 160404 (2005)

[11] N. Barberán, M. Lewenstein, K. Osterloh, and D. Dagnino, Phys. Rev. A 73, 063623 (2006).

[12] J. K. Jain, Phys. Rev. Lett. 63, 199 (1989); J. K. Jain and T. Kawamura, Europhys. Lett. 29, 321 (1995).

[13] N. R. Cooper and N. K. Wilkin, Phys. Rev. B 60, R16279 (1999).

[14] Note that, contrary to the true GS's, the states of Eq. (5) cannot be simply described using local density approximation with a liquid in the trap center, and a crystal on the edge.

[15] M. Kasner and W. Apel, Ann. Phys. (Leipzig) 3, 433 (1994).

[16] M. A. Cazalilla, Phys. Rev. A 67, 063613 (2003).

[17] X.-G. Wen, Quantum Field Theory of Many-Body Systems (Oxford University Press, Oxford, 2004). 